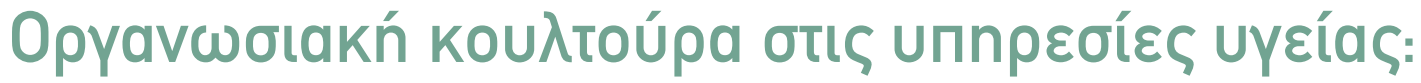

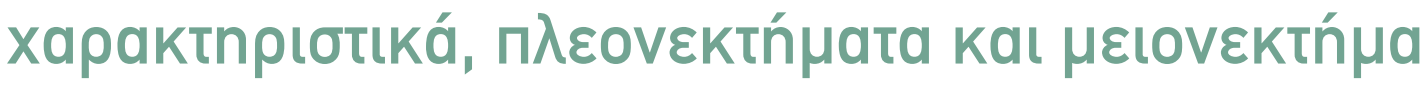

\author{
Ayopítoa Koùoúpn
}

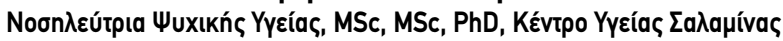

\begin{abstract}
ПЕРIАHWH

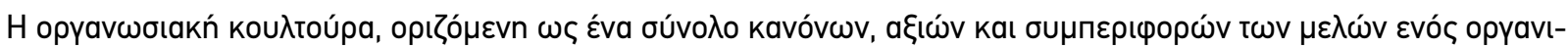

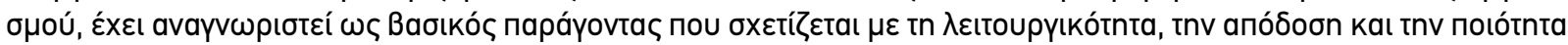

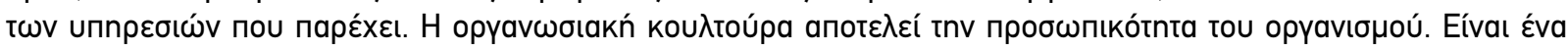

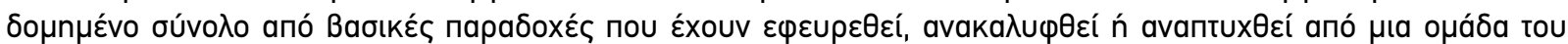

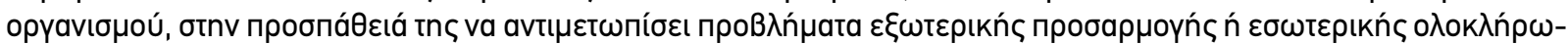

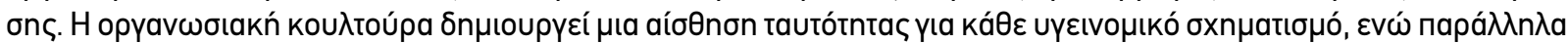

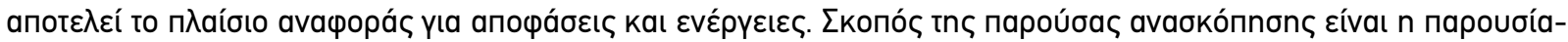

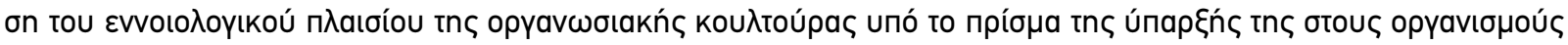

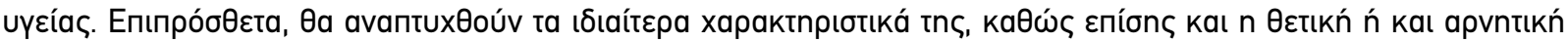

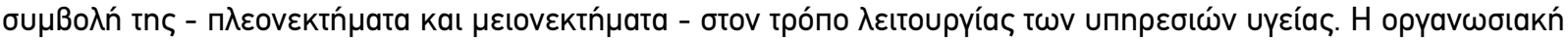

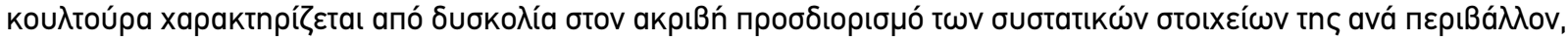

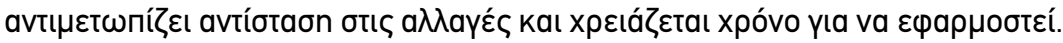

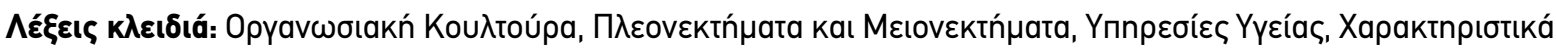




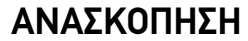

\section{BAEIKA EHMEIA:}

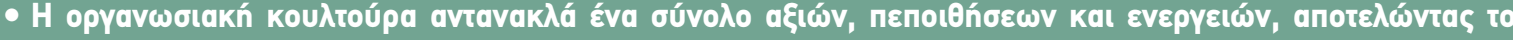
ouvektıkó kpíko yı tnv opá6a uyeías

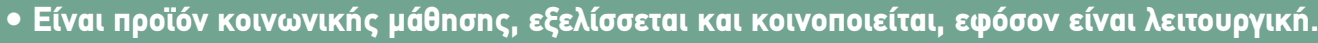

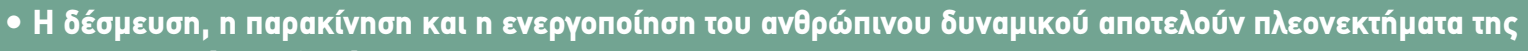
opyavwolakńs kouגtoúpas.

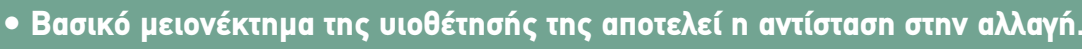

\section{EIIAГQГН}

H

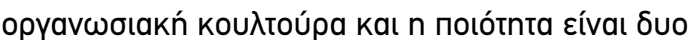

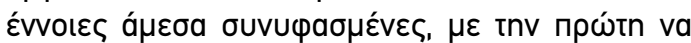

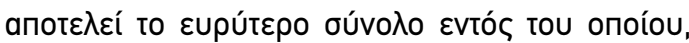

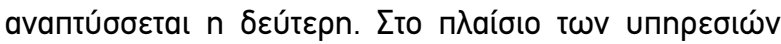

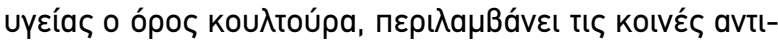

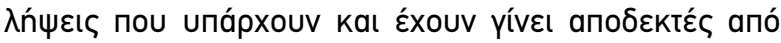

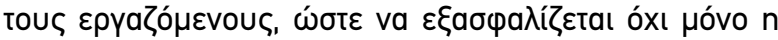

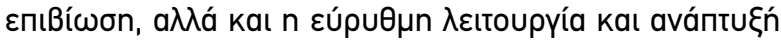
tous (Kápyas 2014).

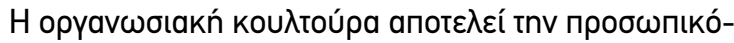

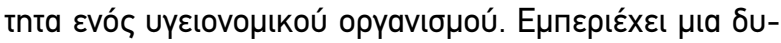

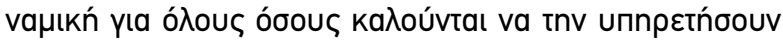

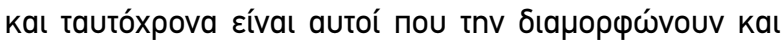

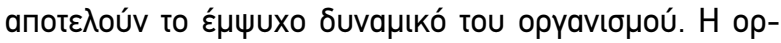

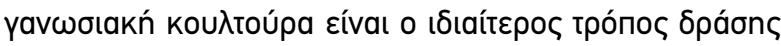

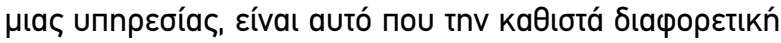

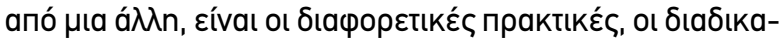

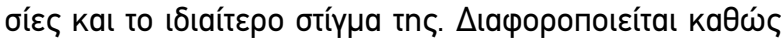

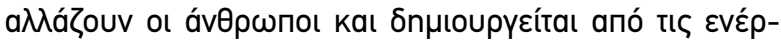

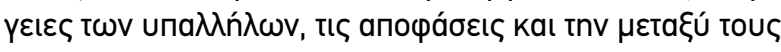

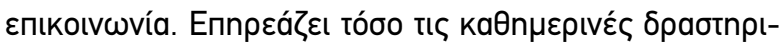

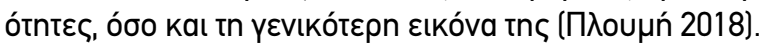

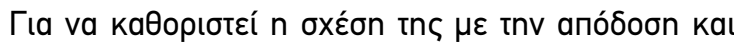

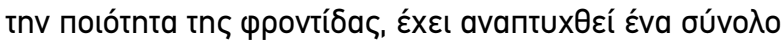

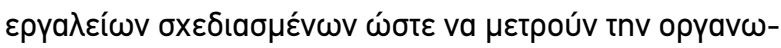

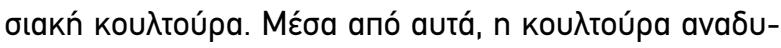

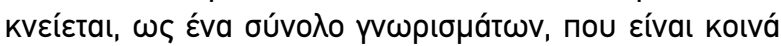

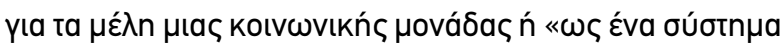

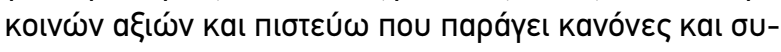

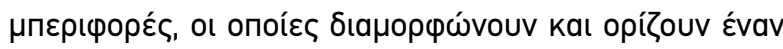

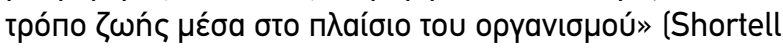

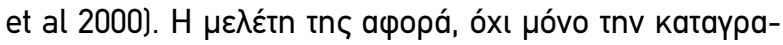

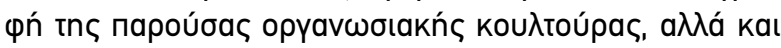

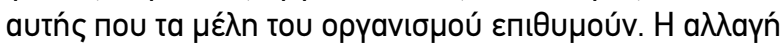

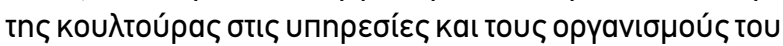

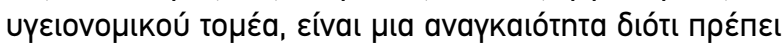

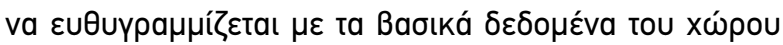

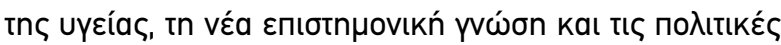

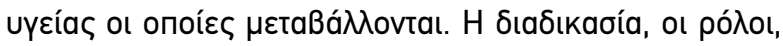

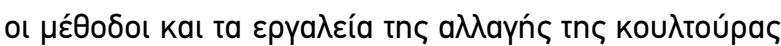

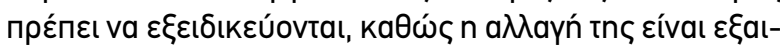

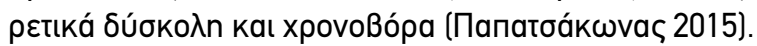

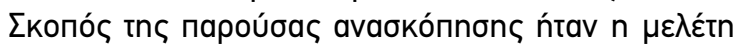

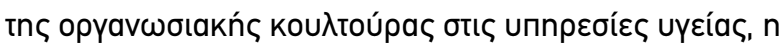

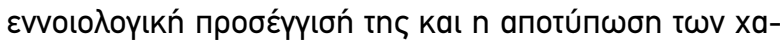

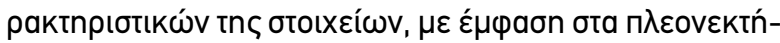

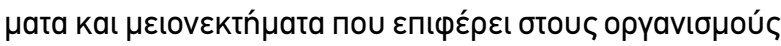
uүzíac.

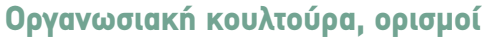

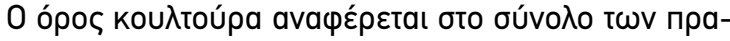

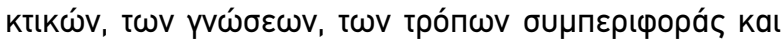

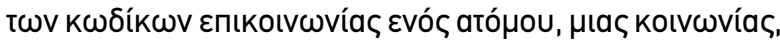

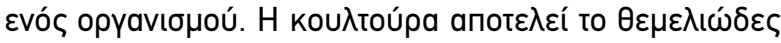

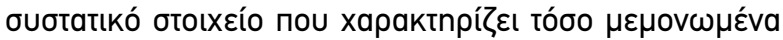

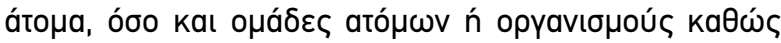

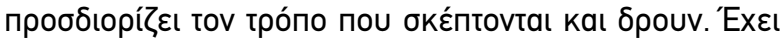

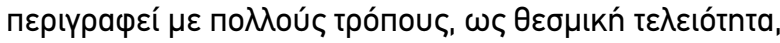

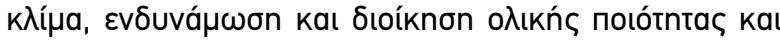

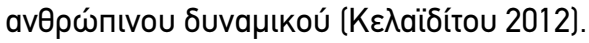

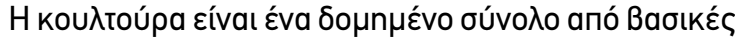

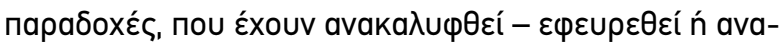

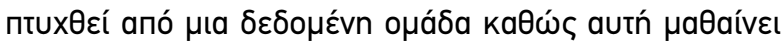

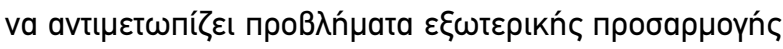

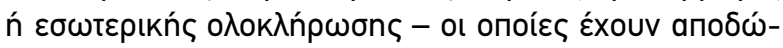

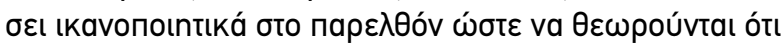

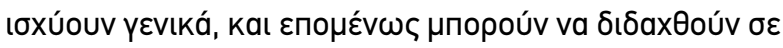

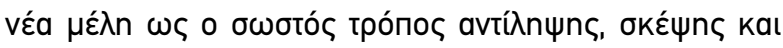

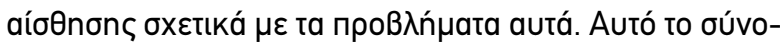

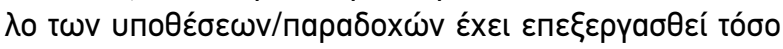

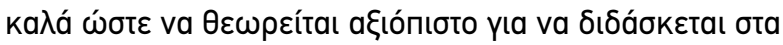

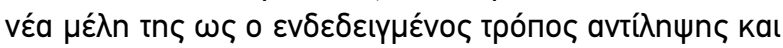

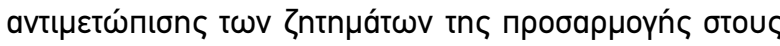

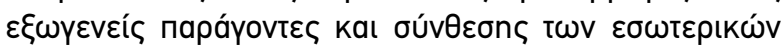
napayóvt $\omega v$ (Schein 1985).

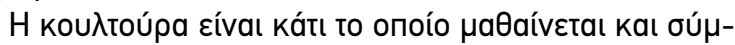

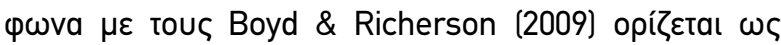

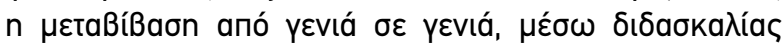

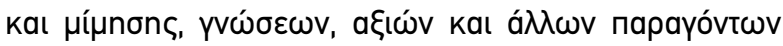




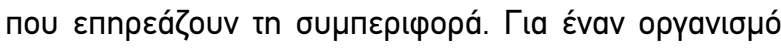

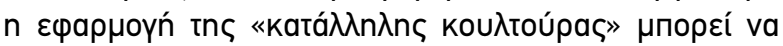

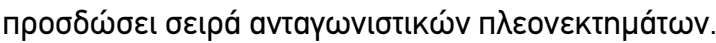

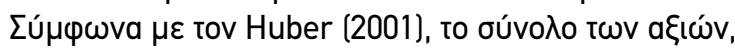

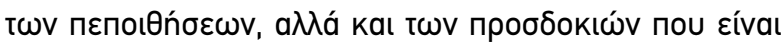
$\varepsilon \cup \rho \varepsilon ́ \omega \varsigma$ бı

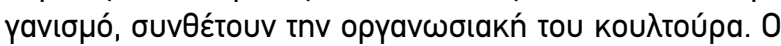

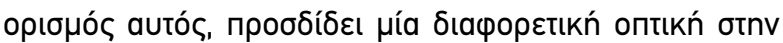

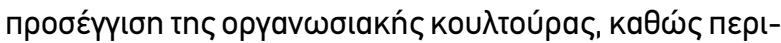

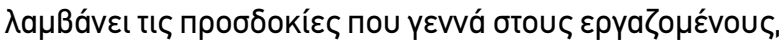

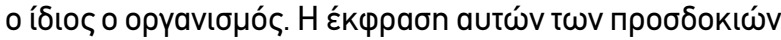

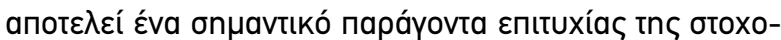

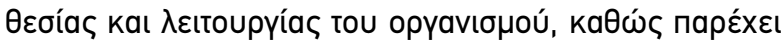

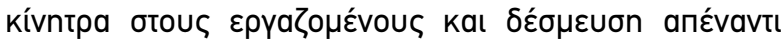

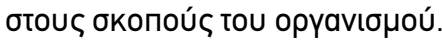

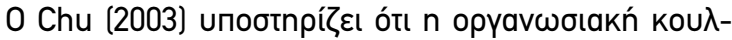

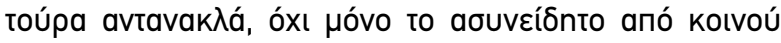

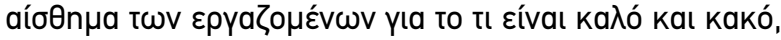

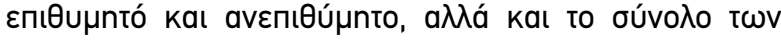

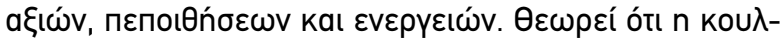

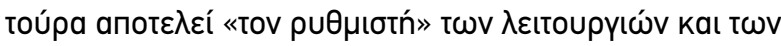

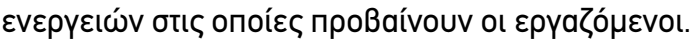

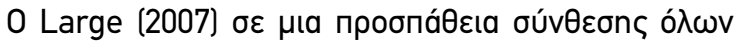

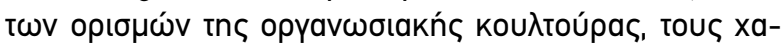

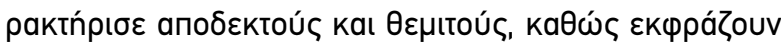

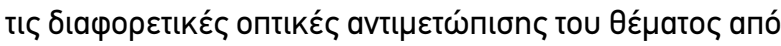

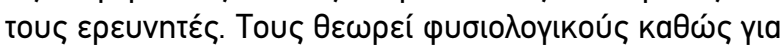

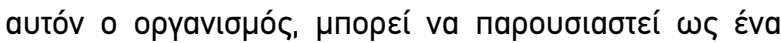

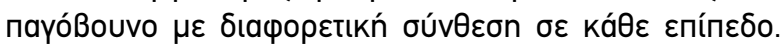

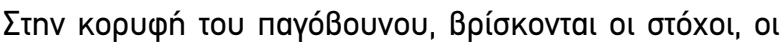

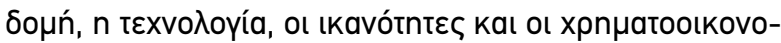

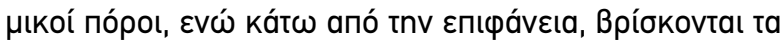

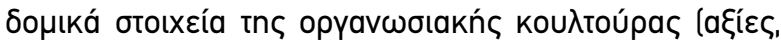

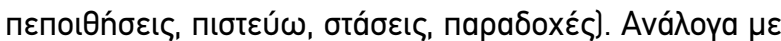

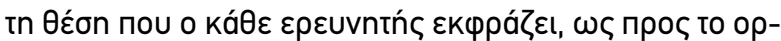

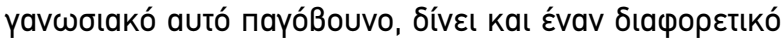

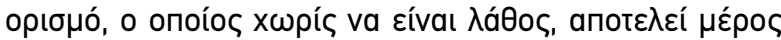

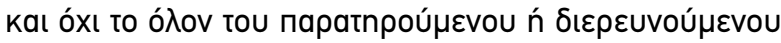
opyaviouoú.

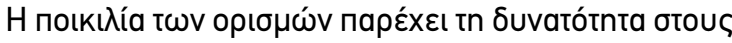

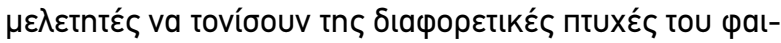

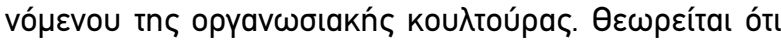

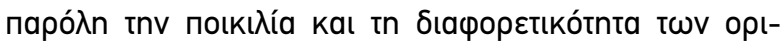

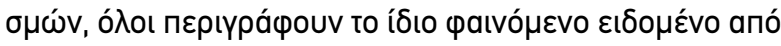

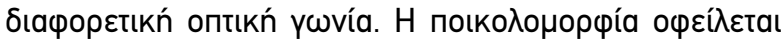

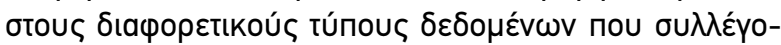

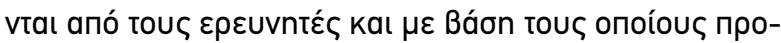

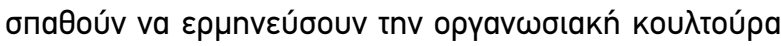

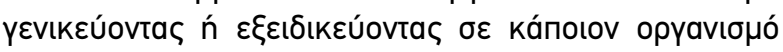
(Kápyac 2014).

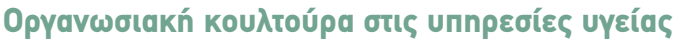

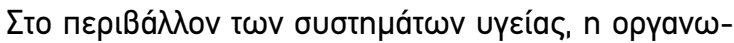

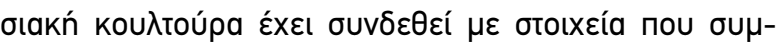

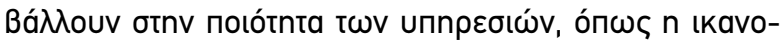

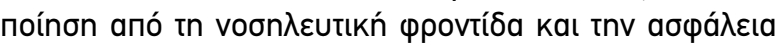

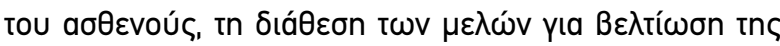

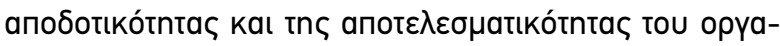

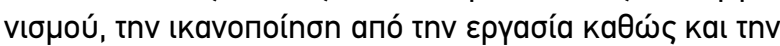

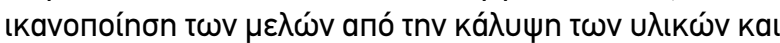

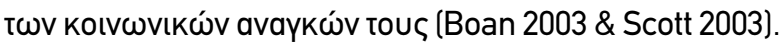

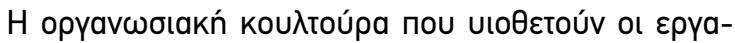

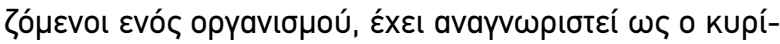

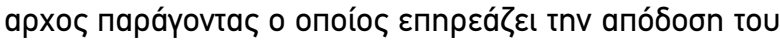

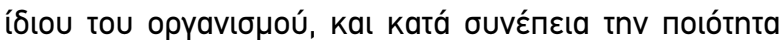

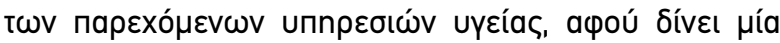

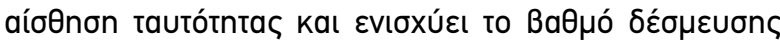

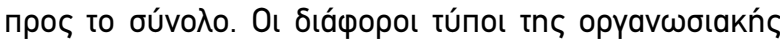

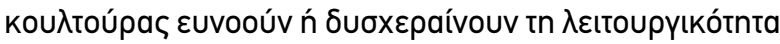

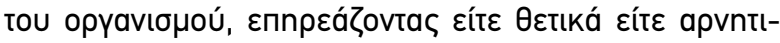

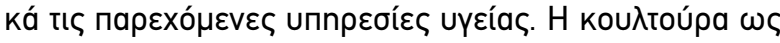

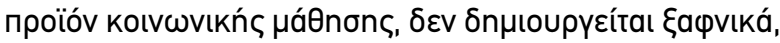

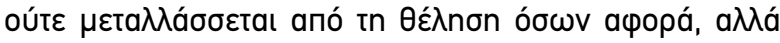

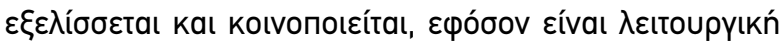
(Пanávns \& Póvtoc 2005).

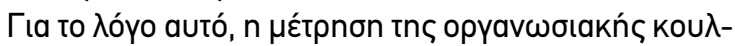

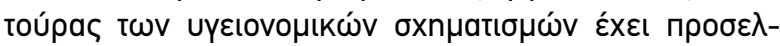

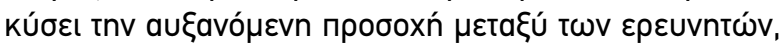

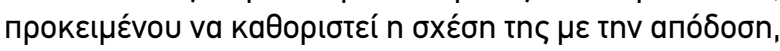

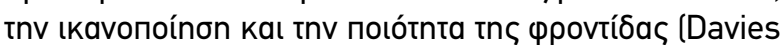
2000 \& Shortell 2000).

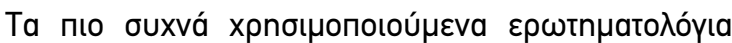

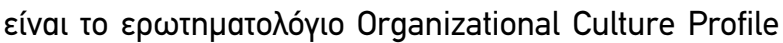

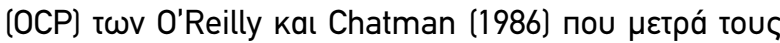

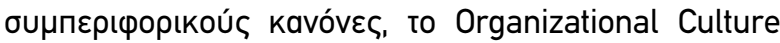

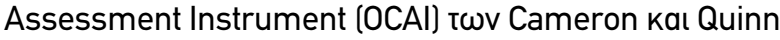

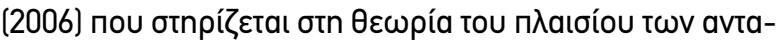

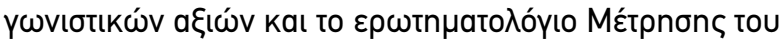

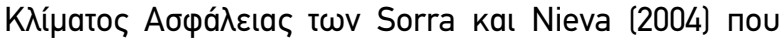

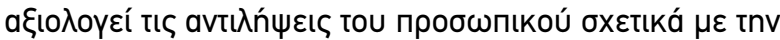

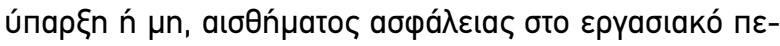

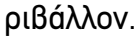

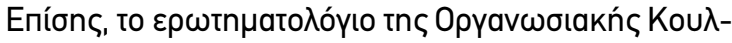

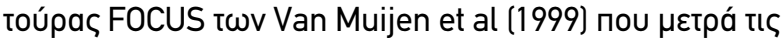

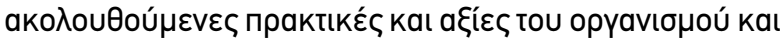

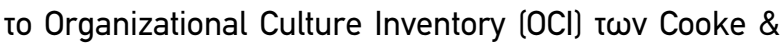

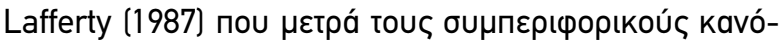

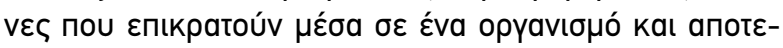

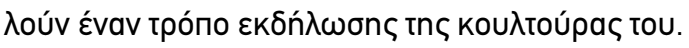

0 Robbins (2001) uпоotnpílદı ótı, n opyavwoıakń

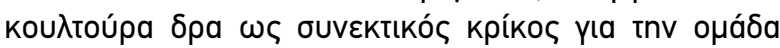

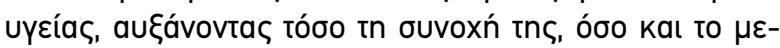

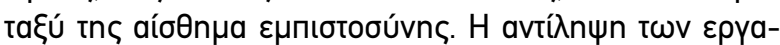

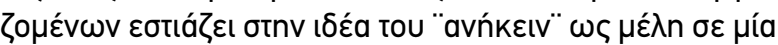

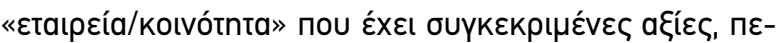

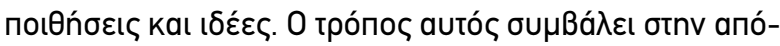




\section{REVIEW}

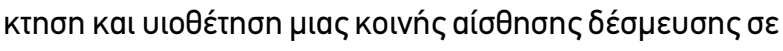

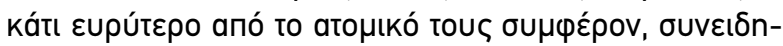

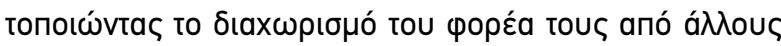

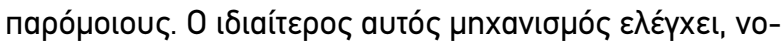

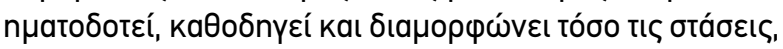

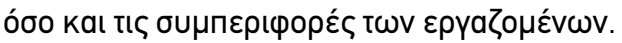

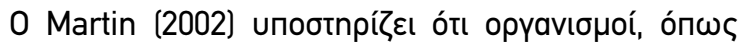

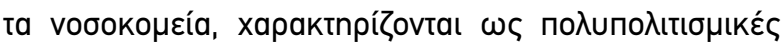

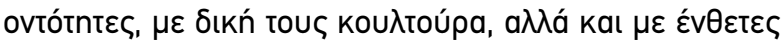

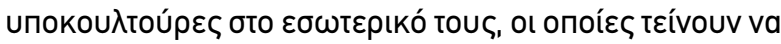

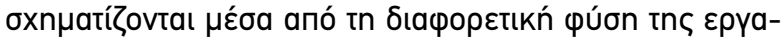

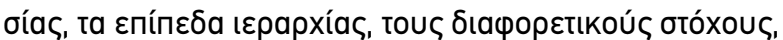

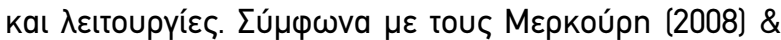

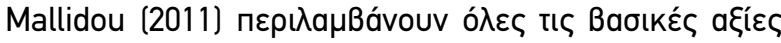

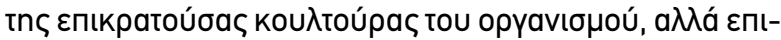

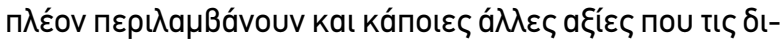

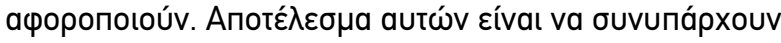

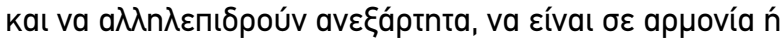

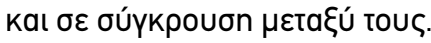

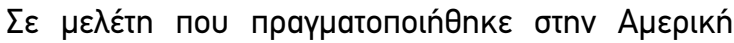

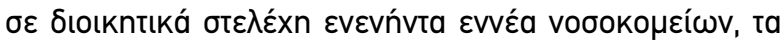

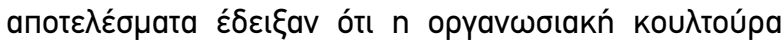

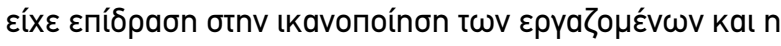

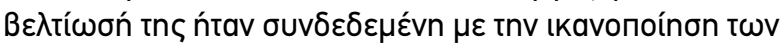

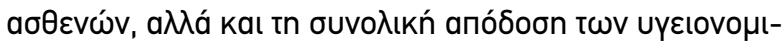

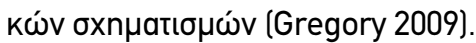

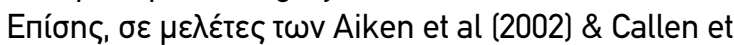

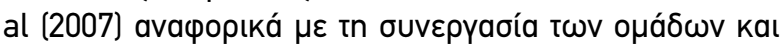

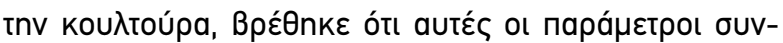

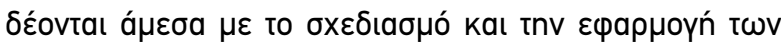

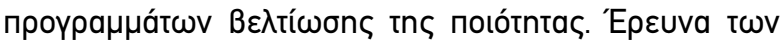

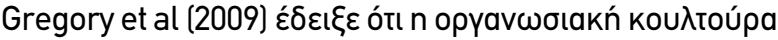

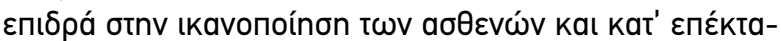

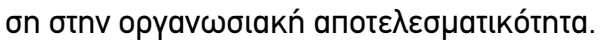

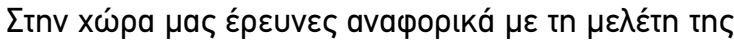

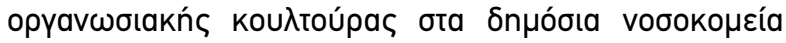

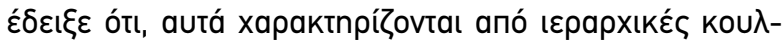

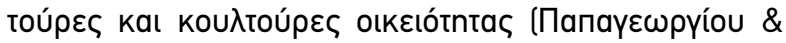

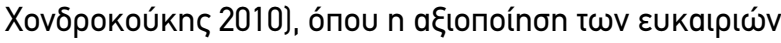

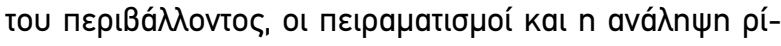

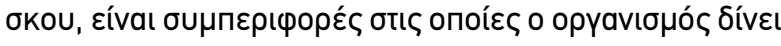

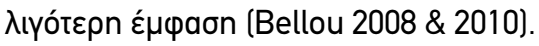

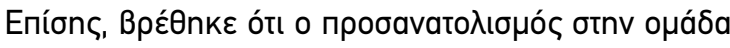

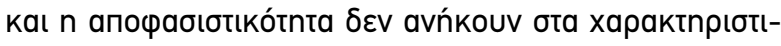

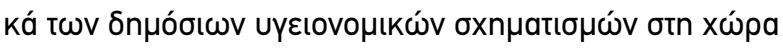

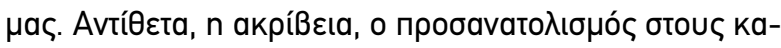

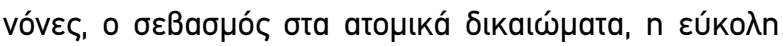

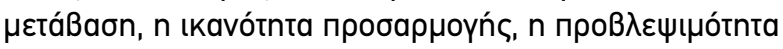

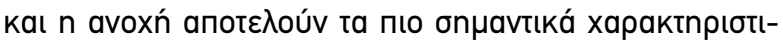

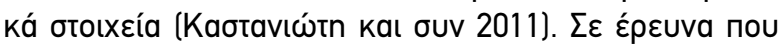

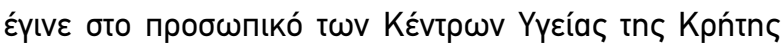

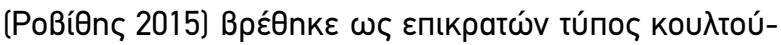

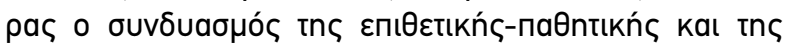

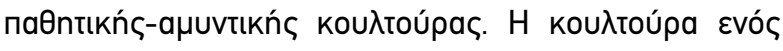

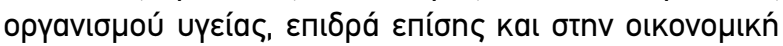

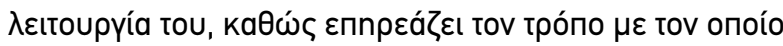

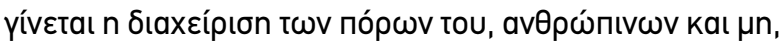

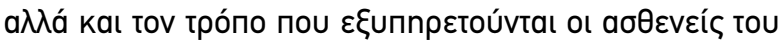

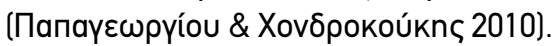

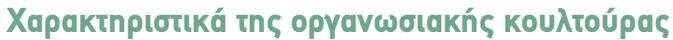

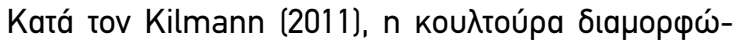

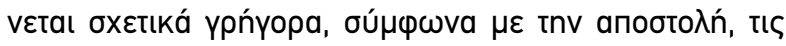

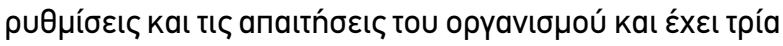
xapakinpiotiká.

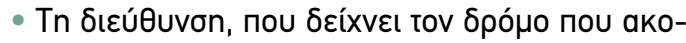

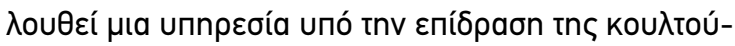

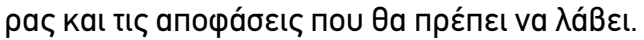

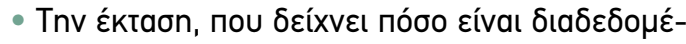

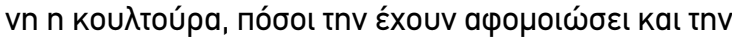

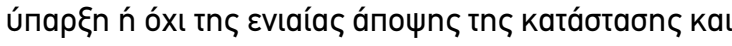

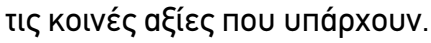

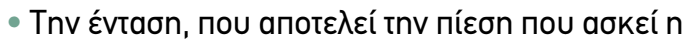

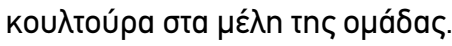

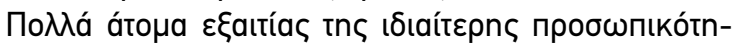

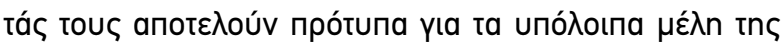

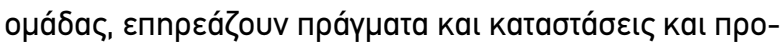

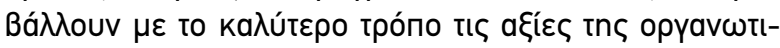

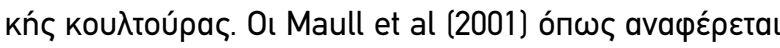

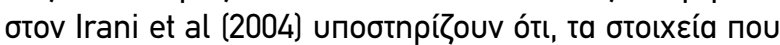

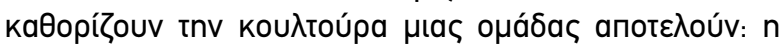

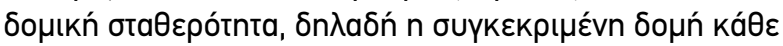

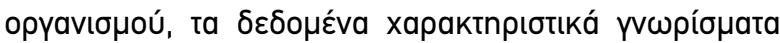

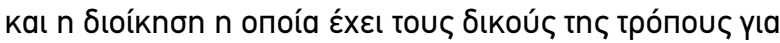

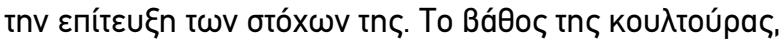

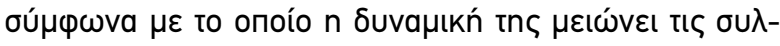

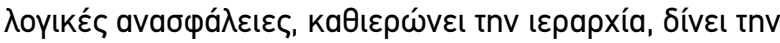

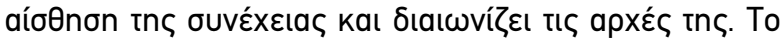

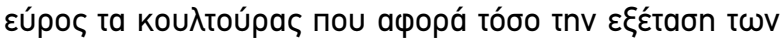

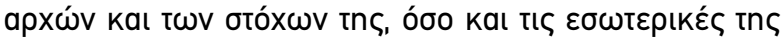

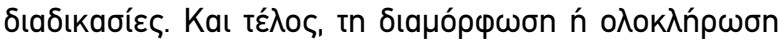

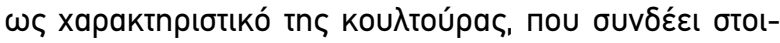

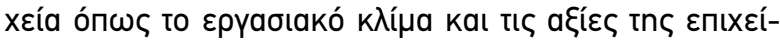

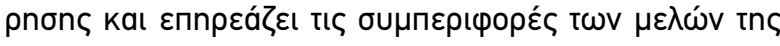

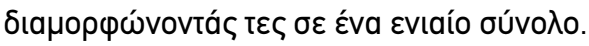

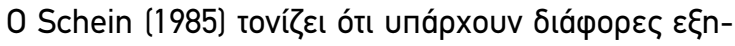

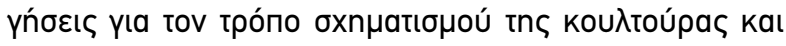

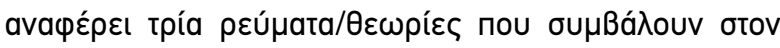

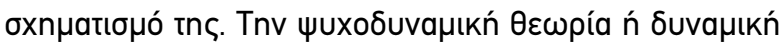

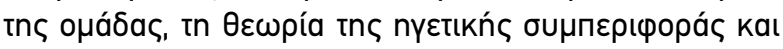

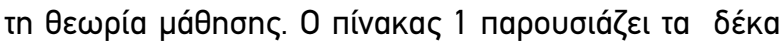

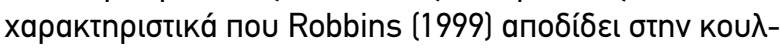
toúpa.

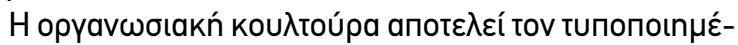

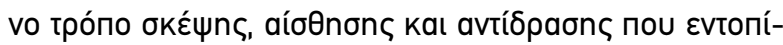

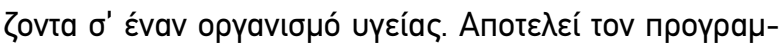




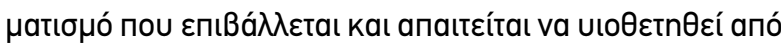

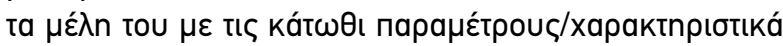
(A $\lambda \varepsilon \xi a ́ v \delta$ pou 2005).

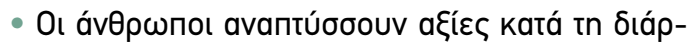

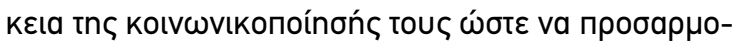

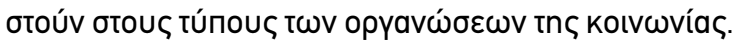

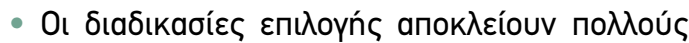

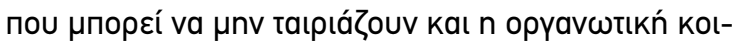

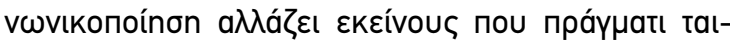

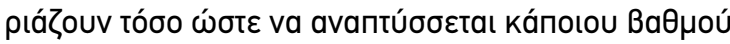

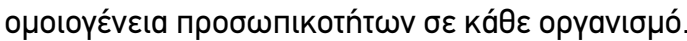

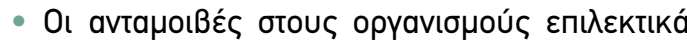

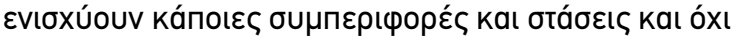

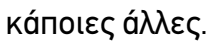

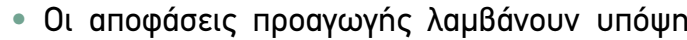

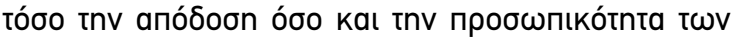
uпочnчí $\omega v$.

Avtíotoıxa o Tsai (2011) unootnpí̧દı ótı n ouvoxń kaı

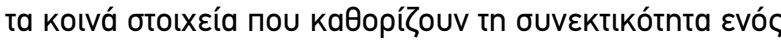

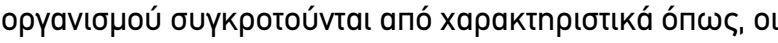

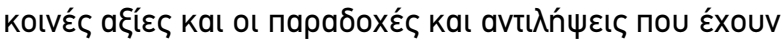

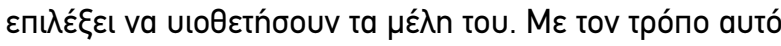

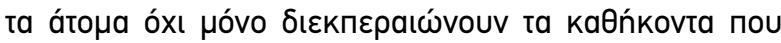

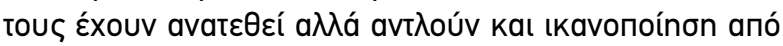

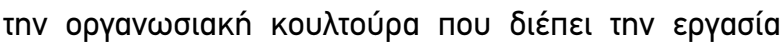
tous.

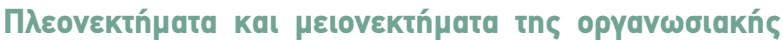

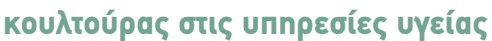

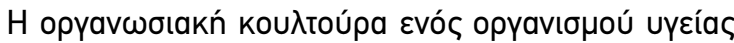

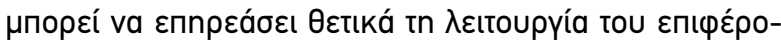

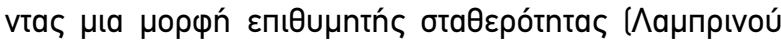

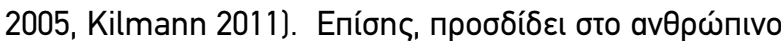

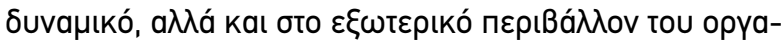

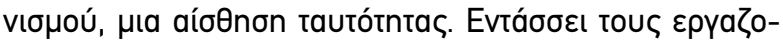

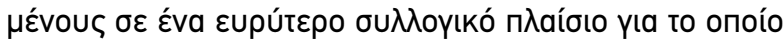

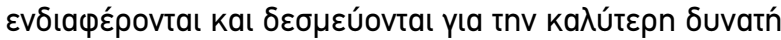

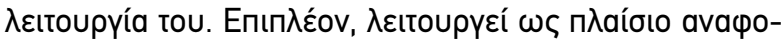

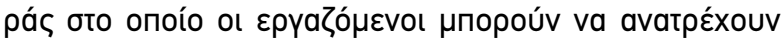

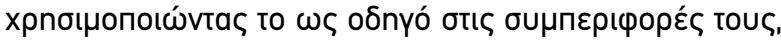

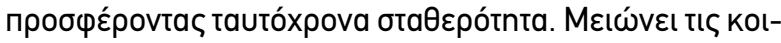

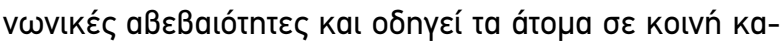

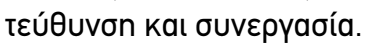

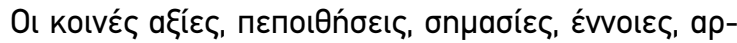

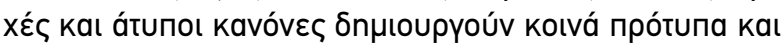

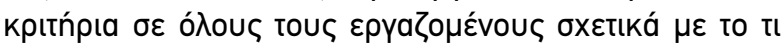

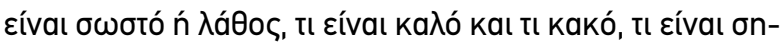

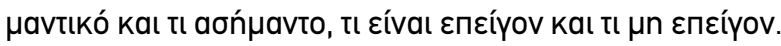

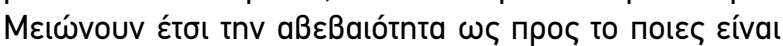

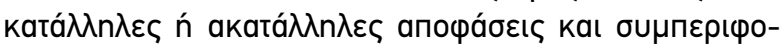

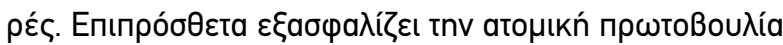

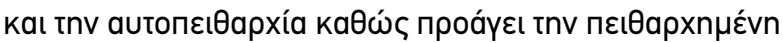

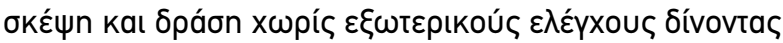

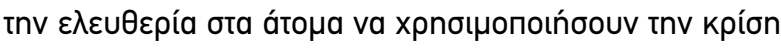

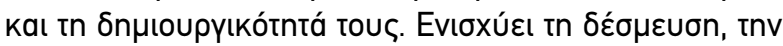

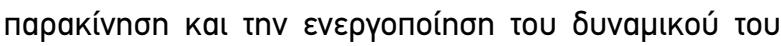

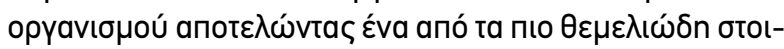
x

¿ú $\mu \omega \omega v a \mu \varepsilon$ tous Martins \& Terblanche (2003), ol

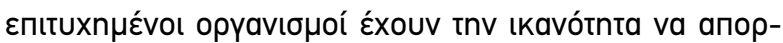

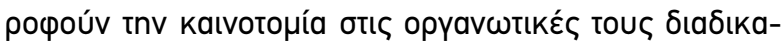

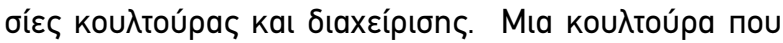

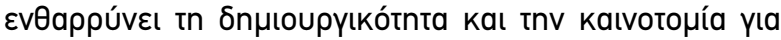

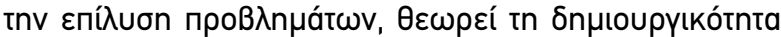

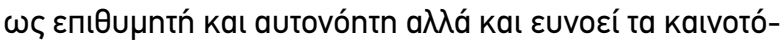

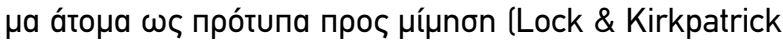
1995).

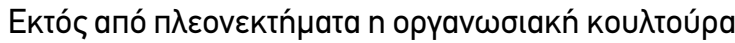

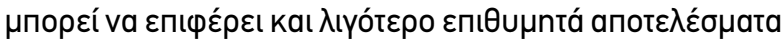

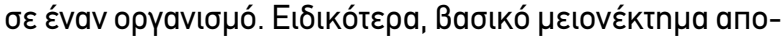

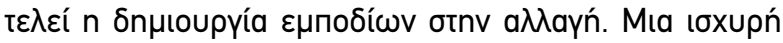

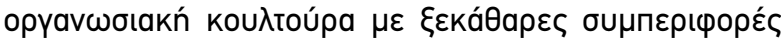

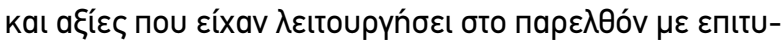

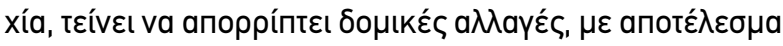

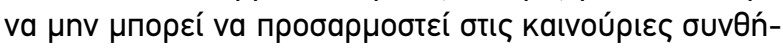

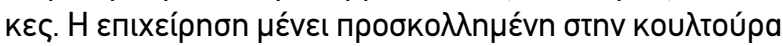

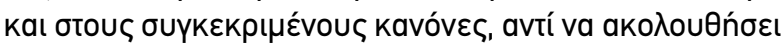

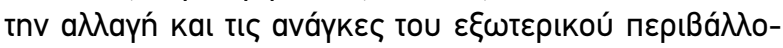

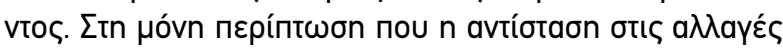

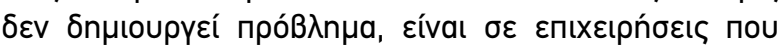

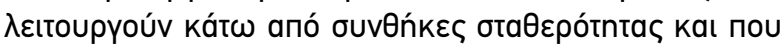

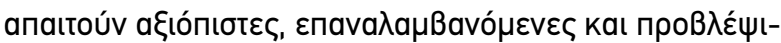

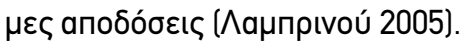

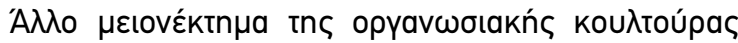

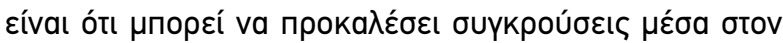

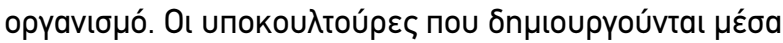

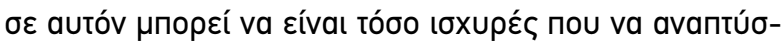

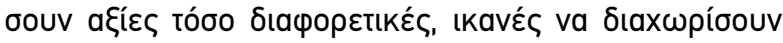

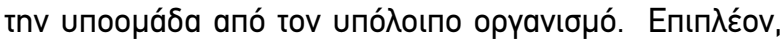

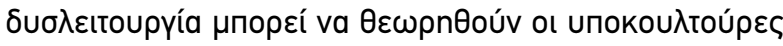

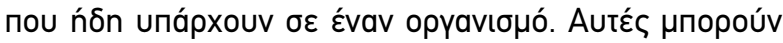

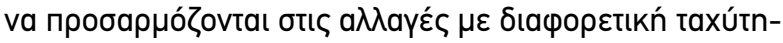

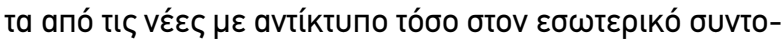

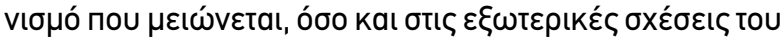
opyavionoú.

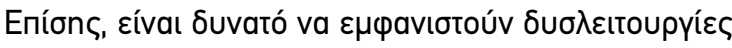

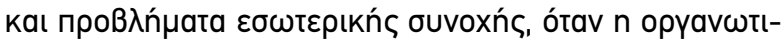

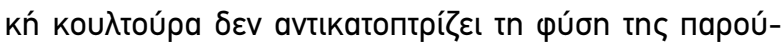

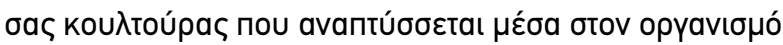

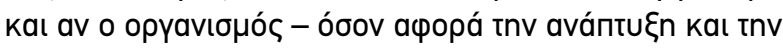

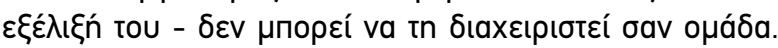

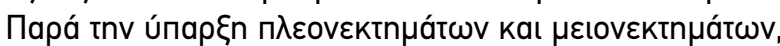

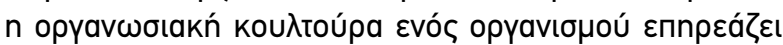

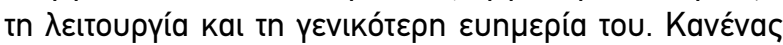

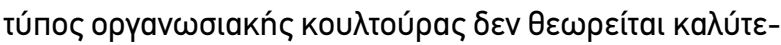

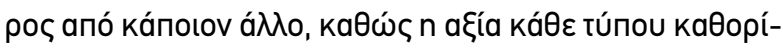




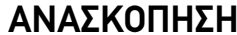

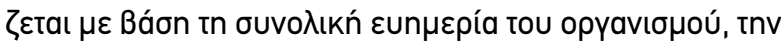
ıkavonoínon $\tau \omega \mathrm{v} \varepsilon \rho \gamma a \zeta o \mu \varepsilon ́ v \omega v$, tnv kкavonoínon $\tau \omega \mathrm{v} \lambda \mathrm{n}$ -

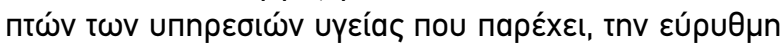

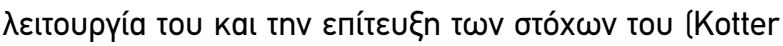
\& Heskett 1992).

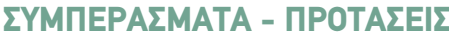

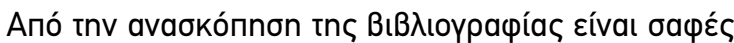

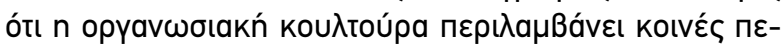

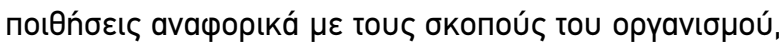

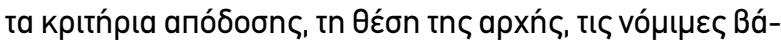

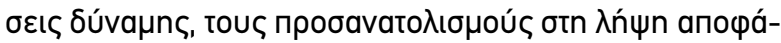

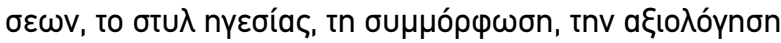

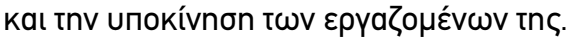

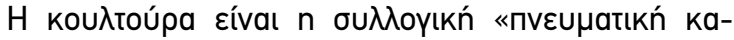

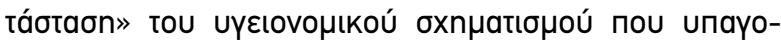

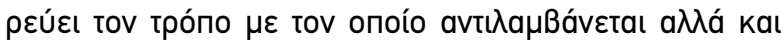

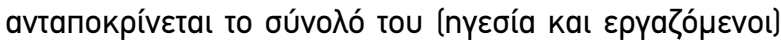

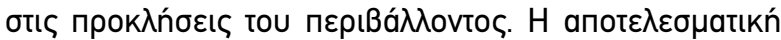

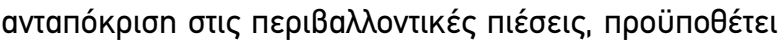

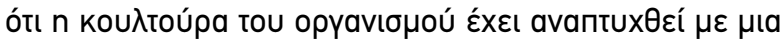

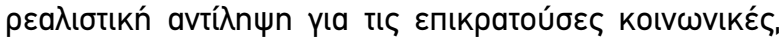

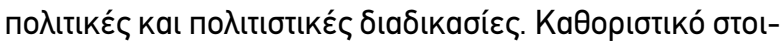

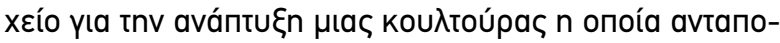

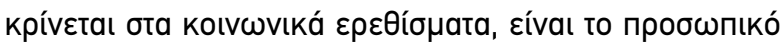

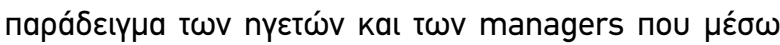

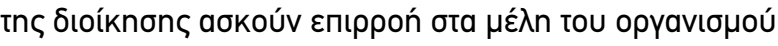
(Chu 2003).

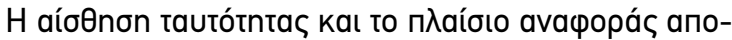

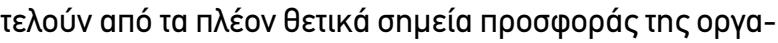

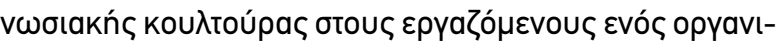

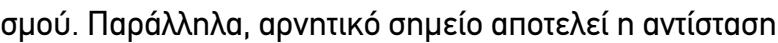

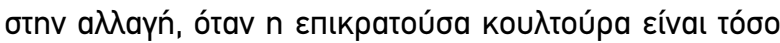

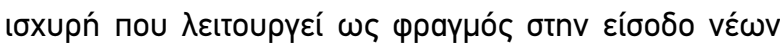

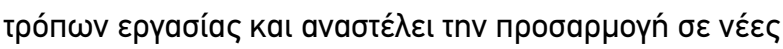

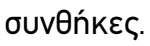

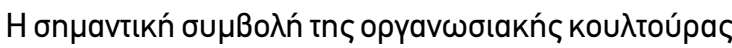

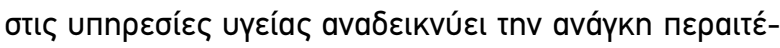

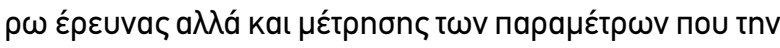

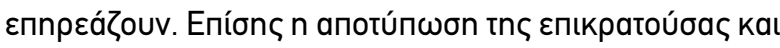

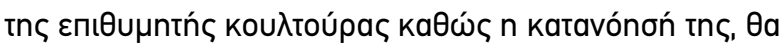

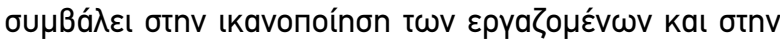

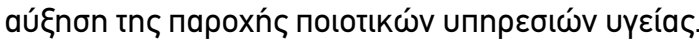

\section{ВІВАІОГРАФІА}

Aiken L.H., Clarke S.P. \& Sloane D.M. (2002). Hospital staffing, organization, and quality of care: Cross-national findings. Nursing Outlook 50(5):187-194.

Bellou V. (2008). Identifying Organizational culture and subcultures within Greek public hospitals. Journal of Health Organization and Management 22(5):496-509.

Bellou V. (2010). Organizational culture as a predictor of job satisfaction: The role of gender and age. Career Development International 15(1):4-19.

Boan D. \& Funderburk F. (2003). Healthcare quality improvement and organizational culture. EASTON MD: Delmarva Foundation.

Callen JL., Braithwaite J \& Westbrook JI. (2007). Cultures in hospitals and their influence on attitudes to, and satisfaction with, the use of clinical information systems. Soc Sci Med 65:635-639.

Cameron K. \& Quinn R. (2006). Diagnosing and changing organizational culture. Jossey-Bass San Francisco, 95:23-30.

Chu K.F. (2003). An organizational culture and the empowerment for change in SMEs in the Hong Kong manufacturing industry," Journal of materials processing technology 139:505.

Cooke R.A. \& Lafferty J.C. (1995). Organizational culture inventory. Plymouth, MI: Human Synergistic International.

Davies H.T.O., Nutley S.M. \& Mannion R. (2000). Organizational culture and quality of health care. Quality in Health Care 9:111-119.

Gregory BT., Harris SG., Armenakis AA. \& Shook CL. (2009). Organizational culture and effectiveness: A study of values, attitudes, and organizational outcomes. J Bus Res 62:673-679.

Huber G. (2001). Transfer of knowledge in knowledge management systems: unexplored issues and suggested studies. European journal of information systems: an official journal of the Operational Research Society 10:72-79.

Irani Z., Beskese A. \& Love P. (2004). Total quality management and corporate culture: constructs of organizational excellence. Elsevier 24(8):643-650.

Karel De Witte., Jaap J. \& van Muijen. (1999). Organizational Culture. Journal European Journal of Work and Organizational Psychology $8(4)$.

Kilmann R.H. (2011). Quantum Organizations: A New Paradigm for Achieving Organizational Success and Personal Meaning. Newport Coast, CA: Kilmann Diagnostics 97-107.

Kotter P. \& Heskett J.L. (1992). Corporate culture and performance. New York: The Free Press.

Large M. (2007). Change, How to Do it and Make it Work, Tools and Techniques for Managing Change. A Joint Working Publication

Lock E.A. \& Kirkpatrick S.A. (1995). Promoting creativity in organizations, in Ford C.M., Gioia D.A. (Eds), Creative Action in Organizations: Ivory Tower Visions \& Real World Voices, Sage, London: $115 \square 20$.

Mallidou AA., Cummings GG., Estabrooks CA. \& Giovannetti PB. (2011). Nurse Specialty subcultures and patient outcomes in acute care hospitals: A multiple-group structural equation modeling. Int $J$ Nurs Stud 48:81-93.

Martin J. (2002). Organizational culture: Mapping the terrain. Thousand Oaks, CA, Sage Publications Inc.

Martins E.C. \& Terblanche F. (2003). Building organizational culture that stimulates creativity and innovation. European Journal of Innovation Management 6(1):64-74.

Maull R., Brown P. \& Cliffe R. (2001). Organizational culture and quality improvement. International Journal of Operations \& Production Management 21: 302-326.

O'Reilly C.A. \& Chatman J. (1986). Organizational commitment and psychological attachment: The effects of compliance, identification, and internalization on prosocial behavior. Journal 
of Applied Psychology 71:492-499.

Robbins S.P. (1999.) Essentials of Organizational Behavior. Pearson College Division. Business \& Economics.

Robbins S.P. (2001). Basic Motivation Concepts, Organizational Behavior. Prentice Hal, New Jersey, 6:155-209

Schein E.N. (1985). Organizational culture and leadership. San Francisco: Jossey-Bass.

Scott T., Mannion R., Davies H. \& Marshall M. (2003).The quantitative measurement of organizational culture in health care: A review of the available instruments. Health Services Res 38:923-945.

Shortell S.M, Jones R.H., Rademaker A.W., Gillies R.R., Dranove D.S., Hughes E.F., Budetti P.P., Reynolds K.S. \& Huang C.F. (2000). Assessing the Impact of Total Quality Management and Organizational Culture on Multiple Outcomes of Care for Coronary Artery Bypass Graft Surgery Patients. Medical Care 38(2):207-17.

Sorra J.S. \& Nieva V.F. (2004). Hospital Survey on Patient Safety Culture. Rockville, MD: Agency for Healthcare Research and Quality.

Tsai Y. (2011). Relationship between organizational culture, leadership behavior and job satisfaction. BMC Health Services Res 14(11):98.

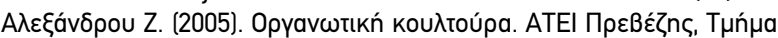

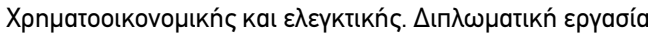

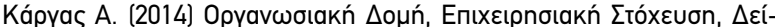

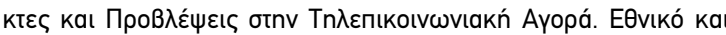

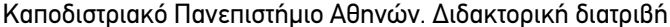

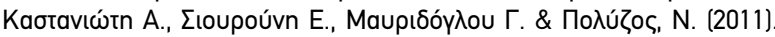

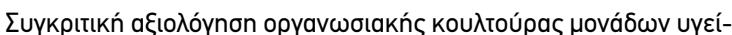
ac. Apxeía EMnvikńs latpıkńs 28(1):70-78.

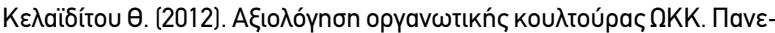

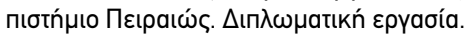

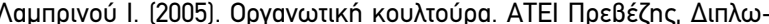

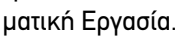

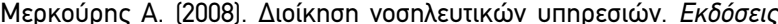
EM

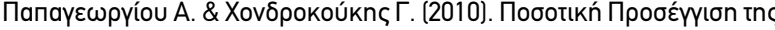

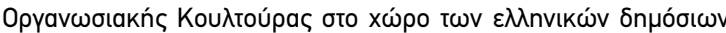

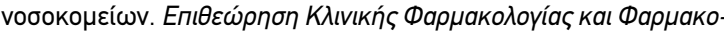
KıVntıKńs 28:269-278.

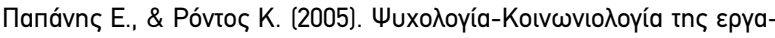

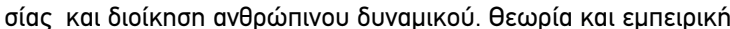

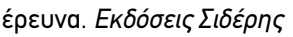

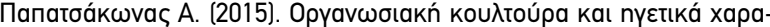

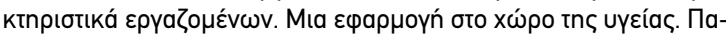

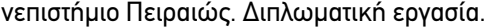

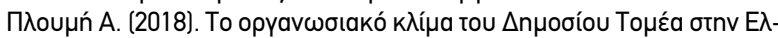

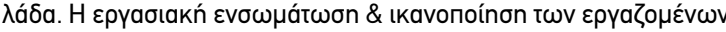

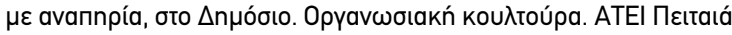

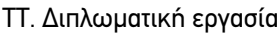

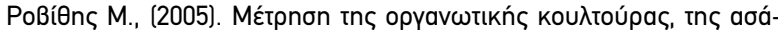

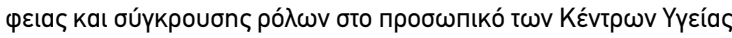

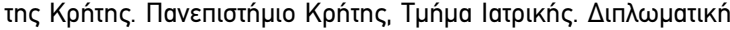
epyaría.

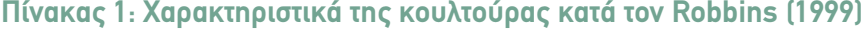

\section{Xapakinplotiká tnৎ̧ kouגtoúpaç}

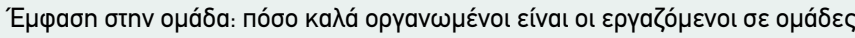

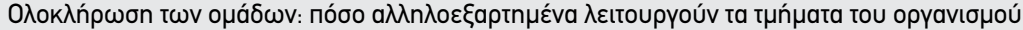

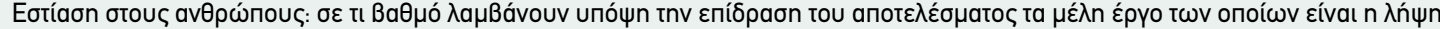

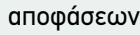

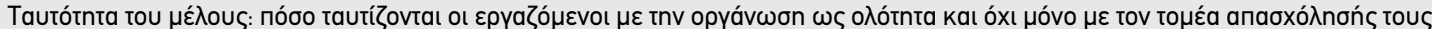

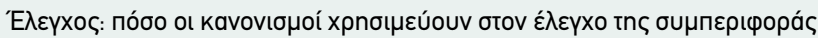

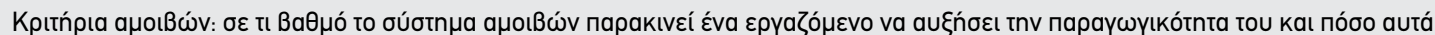

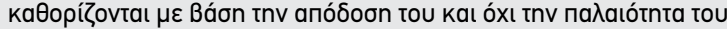

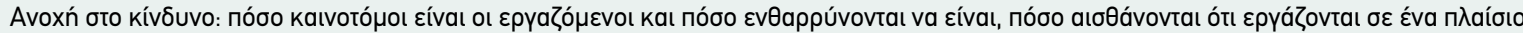
пробтатвutıkó Yıa autoúc

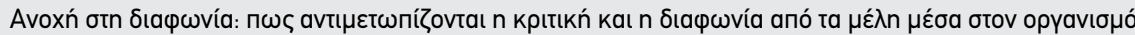

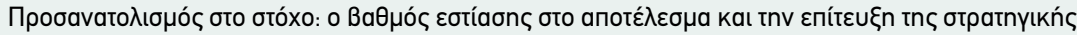

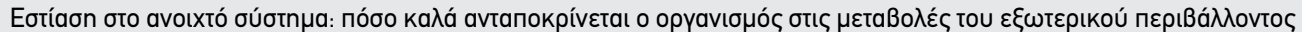




\title{
REVIEW
}

\section{Organizational Culture in Healthcare Services: Characteristics, Advantages and Disadvantages}

\author{
Agoritsa Koulouri
}

RN, RMHN, MSc, MSc, PhD, Health Centre, Salamis

\section{ABSTRACT}

Organizational culture - a system of rules, values and behaviors of an organization's - is a key factor of the functionality, performance and quality of the services it provides. Organizational culture is the personality of the organization. It is a structured set of key assumptions that have been invented, discovered or developed by a team of the organization in order to cope with problems of external adaptation or internal integration. Organizational culture creates a sense of identity for every health formation, while it is serving as a reference frame for decisions and actions. The purpose of this review is to present the conceptual framework of organizational culture in the light of its existence in health organizations. Additionally, the development of its special characteristics as well as its positive and/ or negative contribution (advantages and disadvantages) to the way the health services operate are examined. Organizational culture is characterized by difficulty in identifying its components in regards to every environment, it is facing resistance in change and needs time to be implemented.

Keywords: Advantages and Disadvantages, Health Services, Organizational Culture 